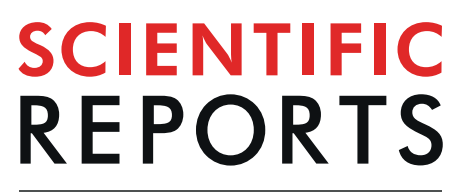

natureresearch

\title{
Cryptogenic stroke and atrial fibrillation in a real-world population: the role of insertable cardiac monitors
}

\author{
Maria Vittoria De Angelis ${ }^{1}$, Vincenzo Di Stefano $\mathbb{1}^{2 *}$, Raffaella Franciotti $\mathbb{D}^{2}$, Nanda Furia ${ }^{3}$, \\ Enrico Di Girolamo ${ }^{3}$, Marco Onofrj ${ }^{2}$ \& Massimiliano Faustino ${ }^{3}$
}

The incidence of atrial fibrillation (AF) in cryptogenic stroke (CS) patients has been studied in carefully controlled clinical trials, but real-world data are limited. We investigated the incidence of AF in clinical practice among CS patients with an insertable cardiac monitor (ICM) placed for AF detection. Patients with CS admitted to our Stroke Unit were included in the study; they received an ICM and were monitored for up to 3 years for AF detection. All detected AF episodes of at least $120 \mathrm{sec}$ were considered. From March 2016 to March 2019, 58 patients (mean age $68.1 \pm 9.3$ years, 67\% male) received an ICM to detect AF after a CS. No patients were lost to follow-up. AF was detected in 24 patients ( $41 \%$, AF group mean age $70.8 \pm 9.4$ years, $62 \%$ male) after a mean time of 6 months from ICM (ranging from 2 days to 2 years) and 8 months after CS (ranging from 1 month to 2 years). In these AF patients, anticoagulant treatment was prescribed and nobody had a further stroke. In conclusion, AF episodes were detected via continuous monitoring with ICMs in $41 \%$ of implanted CS patients. AF in CS patients is asymptomatic and difficult to diagnose by strategies based on intermittent short-term recordings. Therefore, we suggest that ICMs should be part of daily practice in the evaluation of CS patients.

In $20-40 \%$ of ischemic strokes, a definitive cause is not identified, despite extensive evaluation ${ }^{1}$; this condition has been defined as "cryptogenic stroke" $(\mathrm{CS})^{1-6}$. Many hypotheses have arisen to find an explanation for the stroke occurrence, but there is no consensus ${ }^{7}$. Atrial fibrillation (AF) is a well-known cause of ischemic stroke and about $15 \%$ of strokes are attributable to a documented $\mathrm{AF}^{8,9}$. Moreover, $\mathrm{AF}$ is the most common cause of cardioembolism in patients older than $70^{10,11}$. Unfortunately, AF can evade conventional monitoring strategies of patients with acute cerebral ischemia, thus supporting its possible role in $\mathrm{CS}^{12}$. The diagnosis of AF has clinical relevance, as randomized clinical trials have shown that anticoagulation reduces the risk of stroke in patients with $\mathrm{AF}^{13,14}$. However, it is required to document AF to initiate anticoagulant therapy after ischemic stroke and, in the absence of documented AF, antiplatelet agents are recommended ${ }^{15}$. A diagnosis of AF can influence the management of CS, because the presence of AF changes the pharmacological therapy to anticoagulant prescription. Indeed, it has been shown that anticoagulation is superior than antiaggregation in preventing further strokes in patients with $\mathrm{AF}^{16}$. The risk of stroke in patients with $\mathrm{AF}$ can be estimated by the $\mathrm{CHA}_{2} \mathrm{DS}_{2}-\mathrm{VASc}$ score ${ }^{17}$, but, even in the presence of strong suspicion, AF may not be detected in the acute phase of ischemic stroke, due to its paroxysmal and asymptomatic nature ${ }^{18-21}$. For this reason, many strategies have been explored to improve detection of AF, ranging from in-hospital monitoring, serial electrocardiography and Holter monitoring and the use of external events (or loop recorders) or insertable cardiac monitors (ICM) ${ }^{22-24}$.

Recently, it has been demonstrated that ICMs improve the diagnostic sensibility to detect many arrhythmias, especially AF in patients with CS, with detection rates (DR) to $25 \%^{20,22,24}$. Many reports and single-centre studies have appeared, but there was a lack of randomized trials until $2014^{25-28}$. The CRYSTAL-AF study was the first randomized study demonstrating the superiority of ICMs to standard care monitoring in $\mathrm{CS}^{24,29,30}$. However, these trials reflected results in patients with specific conditions, inclusion and exclusion criteria. More recent studies

${ }^{1}$ Department of Neurology, "SS Annunziata" Hospital, Chieti, Italy. ${ }^{2}$ Department of Neuroscience, Imaging and Clinical Sciences, "G. d'Annunzio" University, Chieti, Italy. ${ }^{3}$ Department of Cardiology and Cardiac Surgery, "G. d'Annunzio" University, Chieti, Italy. *email: vincenzo19689@gmail.com 
explored the incidence of AF in real-world cohorts of patients with an ICM implanted after a CS (cardiac event monitor) with a relatively brief follow-up ${ }^{26,31}$. Only a few reports have explored the long-term DR of AF in the CS population $^{32}$.

In the present real-world study, we show the incidence of AF detected by ICM in a cohort of 58 CS consecutively recruited patients with 3 years of follow-up. These data reinforce literature data on long-term monitoring (up to 2 years) with the use of ICMs in CS patients. The wide use of ICMs in clinical practice could significantly enhance the detection of $\mathrm{AF}$ and improve the prognosis, allowing early initiation of anticoagulant therapy, in a relevant percentage of patients with CS.

\section{Methods}

The study was performed according to the declaration of Helsinki and its later amendments, and it was approved by the ethical committee of the "SS Annunziata" hospital of Chieti. All patients included in the study consented to the use of their data for research purposes and signed informant consent for study participation.

Patient population. We included all patients admitted to the Neurological Clinic of "SS Annunziata" hospital of Chieti, which received an ICM after a cryptogenic stroke. A stroke was classified as "cryptogenic" if extensive testing failed to reveal a clear aetiology. The workup included: complete neurological examination and $\mathrm{CHA}_{2} \mathrm{DS}_{2}$-VASc score, 24-hours ECG monitoring, transthoracic echocardiography, screening for thrombophilic states (under 55 years of age), transcranial and neck Doppler ultrasound, magnetic resonance angiography, computed tomography angiography of the head and neck. Each patient was monitored for at least 4 months after device insertion. Then, patients were divided into two groups based on the presence (AF group) or absence (no-AF group) of AF (which was recorded by ICM after stroke).

ICM device. The ICM device (Reveal LINQ, Medtronic) is a small cardiac monitor that is inserted into the subcutaneous tissue over the heart. This device detects and records automatically AF, irrespective of heart rate or symptoms ${ }^{32}$. ICM recordings were monitored constantly to detect possible ECG anomalies during the study. The Medtronic CareLink ${ }^{\mathrm{TM}}$ Network was used to transmit the device data remotely. The device will automatically initiate wireless transmission every night for episodes recorded during the prior day. Patients may also initiate a full transmission of all data contained within the device's memory at any time.

Data were collected in the One Hospital ClinicalService project (Clinical Trial Registration Information: http://clinicaltrials.gov/ct2/show/NCT01007474), an observational medical care project that periodically provides our site with information and analysis of clinical and device data regarding our stroke patient population wearing a Medtronic ICM. Main aim of the project is to help healthcare providers in improving patient outcomes and understanding of their patients' care or patients' management. The project was approved by Hospital Medical Director and conformed to the Declaration of Helsinki. All patients provided their informed consent for data collection and analysis.

Follow-up visits were scheduled at 1 month and every 3 months thereafter until study closure, with unscheduled visits in the event of symptom occurrence or after the transmission of ICM data, if advised by the investigator.

$\mathrm{AF}$ was defined as an episode of irregular heart rhythm, without detectable $\mathrm{P}$ waves, lasting more than $120 \mathrm{sec}-$ onds. An expert cardiologist (FM) validated all recorded AF episodes. Only confirmed AF episodes were included in the analysis.

Statistical analysis. We reported continuous variables as mean with standard deviation and categorical variables as numbers (percentage). We compared categorical variables among AF and no-AF groups with the Chi-square test and continuous variables with the Mann-Whitney test. We performed all tests using SPSS (v22) and established the level of significance at the 0.05 level.

Ethical standards. the study was performed in accordance with the ethical standards laid down in the 1964 Declaration of Helsinki and its later amendments, and it was approved by the local ethical committee of Chieti. All patients gave their informed consent prior to their inclusion in the study.

\section{Results}

Patients. Table 1 summarizes demographic and follow-up data from our patients. From March 2016 to March 2019,58 patients (mean age $68.1 \pm 9.3$ years, $67 \%$ male) were consecutively enrolled and received an ICM to detect AF after a CS. Among all 58 patients, the most frequent risk factors of stroke were hypertension $(67 \%$ of patients), hypercholesterolemia (62\%), diabetes (26\%), smoking (16\%) and previous stroke (14\%, Table 1). No patients had heart failure. The Chi-square test showed that hypertension, diabetes, smoking, hypercholesterolemia, coronary artery disease, baseline and discharge NIHSS were not significantly different between the $\mathrm{AF}$ and no-AF group; the only exceptions were previous stroke (26\% in the AF and $6 \%$ in the no-AF group, $\mathrm{p}=0.025)$, and $\mathrm{CHA}_{2} \mathrm{DS}_{2}$-VASc score $(4.9 \pm 1.2$ in the $\mathrm{AF}$ and $4.1 \pm 1.0$ in the no-AF group, $\mathrm{p}=0.035)$.

ICM implantation and follow-up. ICM was implanted after a mean time of $83 \pm 74$ days (ranging from 3 to 453 days) from CS (no significant difference among groups, Table 1). No patients were lost to follow-up. All patients were followed-up for a mean of $906 \pm 288$ days after device insertion (no significant difference among groups). Patients of the no-AF group were monitored for a mean time of 24 months after CS ranging from 6 months to 3.5 years, and a mean time of 21 months after ICM implantation ranging from 3 months to 3 years. In the no-AF group the follow-up time from ICM implantation was lower than 6 months in 7 patients (18\%), 12 months in 10 patients (26\%), 18 months in 5 patients (13\%) and 24 months in 8 patients (21\%). Eight patients $(21 \%)$ of the no-AF group were followed for a time greater than 2 years. All patients tolerated ICM and none required it to be removed. 


\begin{tabular}{|l|l|l|l|l|}
\hline & Total $(\mathbf{n}=\mathbf{5 8})$ & AF group $(\mathbf{n}=\mathbf{2 4})$ & No-AF group $(\mathbf{n}=\mathbf{3 4})$ & P value \\
\hline Age, years & $68.1 \pm 9.3$ & $70.8 \pm 9.4$ & $66.2 \pm 8.6$ & 0.06 \\
\hline Male, $\mathrm{n}(\%)$ & $39(67 \%)$ & $15(62 \%)$ & $24(71 \%)$ & 0.91 \\
\hline Time to ICM (from CS), days & $83 \pm 74$ & $68 \pm 59$ & $94 \pm 86$ & 0.38 \\
\hline Time to AF (from CS, days) & NA & $257.8 \pm 182.9$ & NA & $/$ \\
\hline Time of follow-up (from ICM, days) & $906 \pm 288$ & $938 \pm 268$ & $885 \pm 301$ & 0.68 \\
\hline Time of follow-up (from CS, days) & $989 \pm 270$ & $1006 \pm 250$ & $977 \pm 285$ & 0.14 \\
\hline CHA2DS2-VASc score & $4.4 \pm 1.4$ & $4.9 \pm 1.2$ & $4.1 \pm 1.0$ & $0.035^{*}$ \\
\hline Hypertension & $39(67 \%)$ & $17(70 \%)$ & $22(65 \%)$ & 0.90 \\
\hline Diabetes & $15(26 \%)$ & $5(22 \%)$ & $10(29 \%)$ & 0.051 \\
\hline Smoking & $9(16 \%)$ & $3(13 \%)$ & $6(17 \%)$ & 0.81 \\
\hline Hypercholesterolemia & $36(62 \%)$ & $13(54 \%)$ & $23(68 \%)$ & 0.94 \\
\hline Heart failure & 0 & 0 & 0 & $/$ \\
\hline Coronary artery disease & $7(12 \%)$ & $4(17 \%)$ & $3(9 \%)$ & 0.74 \\
\hline Previous stroke & $8(14 \%)$ & $6(26 \%)$ & $2(6 \%)$ & $0.02)^{*}$ \\
\hline Baseline NIHSS score & $4.40 \pm 2.14$ & $3.79 \pm 1.50$ & $4.84 \pm 2.56$ & 0.63 \\
\hline Discharge NIHSS score & $1.77 \pm 1.60$ & $1.83 \pm 1.23$ & $1.72 \pm 1.82$ & 0.15 \\
\hline Echocardiography: EF, $\%$ & $61.55 \pm 3.96$ & $59.3 \pm 5.0$ & $62.9 \pm 3.0$ & 0.18 \\
\hline $\begin{array}{l}\text { Echocardiography: left atrial } \\
\text { volume index (LAVI), ml } / \mathrm{m}^{2}\end{array}$ & $33.45 \pm 9.94$ & $38.7 \pm 9.1$ & $30.1 \pm 8.0$ & $0.012^{*}$ \\
\hline
\end{tabular}

Table 1. Demographic data, $\mathrm{CHAD}_{2} \mathrm{VASC}_{2}$ score, risk factors, stroke severity, echocardiography measures and temporal monitoring for $\mathrm{AF}$ in all patients and in $\mathrm{AF}$ and no-AF group. Data are shown as means \pm standard deviation for continuous variables or numbers (percentage) for categorical variables. AF, atrial fibrillation; ICM, insertable cardiac monitor; CS, cryptogenic stroke; NA not applicable; EF, ejection fraction. * ${ }^{*}<0.05$.

Freedom from AF

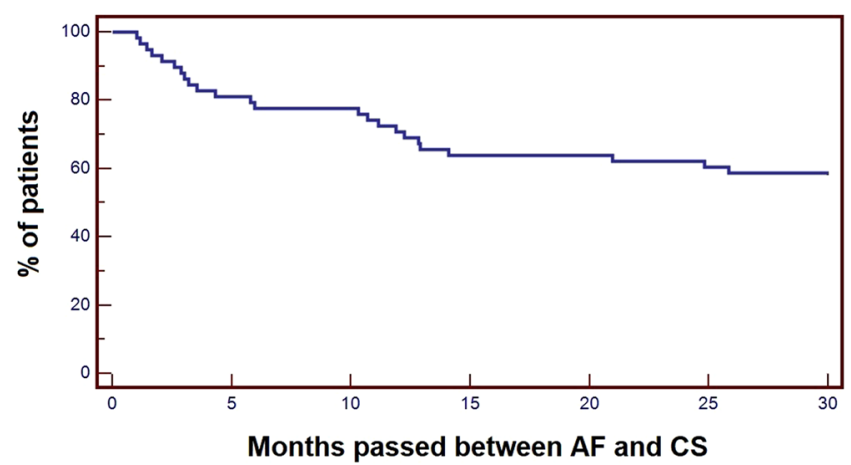

Figure 1. Kaplan-Meier cumulative curve for all patients when the time passed between the occurrence of AF and the CS is used as the survival time variable. AF, atrial fibrillation; CS, cryptogenic stroke.

AF detection. AF was detected in 24 patients ( $41 \%, 62 \%$ male), which received the ICM after a mean time of 68 days (ranging from 6 to 317 days) from CS. Mean age was $70.8 \pm 9.4$ years for AF group and $66.2 \pm 8.6$ years for no-AF group (71\% male). No-AF group was younger $(\mathrm{p}=0.06)$ than AF group. The no-AF group received the ICM after a mean time of $94 \pm 86$ days from CS. The AF and no-AF group were not significantly different for the elapsed time between the CS and the ICM implantation $(p=0.38)$.

AF was detected after a mean time of 6 months from ICM (ranging from 2 days to 2 years) and 8 months after CS (ranging from 1 month to 2 years). Specifically, AF was detected within 6 months after CS in 13 patients (54\% of AF group, 22.4\% among all CS), between 6-12 months after CS in 5 patients (20.8\% in AF group, $8.6 \%$ among all CS), between 13-18 months after CS in 3 patients (12.5\% in AF group, 5.2\% among all CS). Only 3 patients ( $12.5 \%$ in the AF group, 5.2\% among all CS) showed AF after 21, 25 and 26 months after CS respectively. Figure 1 shows the Kaplan-Meier cumulative curve for AF when the time passed between the occurrence of AF and the CS is used as the survival time variable.

AF characteristics and burden. AF was asymptomatic in all patients in the AF group. Twenty-four patients had single or multiple episodes of AF; ventricular arrhythmia and atrioventricular blocks were reported in one and two patients respectively. 
In the AF group we registered a mean overall AF burden of $32 \pm 52$ days and a mean daily AF burden of $57 \pm 82$ minutes for patient. The maximum duration of AF episode was $681 \pm 635$ minutes (range from 2 minutes to 4 days). We excluded a patient from analysis because he developed persistent AF.

Echocardiography. All patients were evaluated through transthoracic echocardiography; there was no significant difference among ejection fraction among groups (Table 1), but echocardiography demonstrated higher left atrial volume index (LAVI) in the AF $\left(38.7 \pm 9.1 \mathrm{ml} / \mathrm{m}^{2}\right)$ compared to the no-AF group $\left(30.1 \pm 8.0 \mathrm{ml} / \mathrm{m}^{2}\right.$, $\mathrm{p}=0.0119)$.

Anticoagulant treatment and outcome. In all AF patients, anticoagulant treatment was prescribed and nobody had another stroke. However, in the no-AF group, one patient ( $3 \%$ of the no-AF group, $1.7 \%$ among all CS) had further strokes after six months from the first CS.

\section{Discussion}

The incidence of AF in CS patients has been studied in carefully controlled clinical trials, but real-world data are limited. A significant number of patients with CS can suffer from AF which is not detected during the standard diagnostic procedures. Indeed, AF may often be paroxysmal and asymptomatic; many patients with AF have long period of sinus rhythm, making short duration non-continuous monitoring suboptimal for excluding $\mathrm{AF}^{18,33}$.

There are several non-invasive strategies, in fact, for detection of AF after stroke ${ }^{34}$, including in-hospital monitoring $^{35}$, serial electrocardiography ${ }^{36}$, Holter monitoring, the use of external events (or loop recorders) ${ }^{22,23}$ and long-term outpatient monitoring ${ }^{37}$. Studies that evaluated holter monitoring ranging from 24 to 72 hours, have a low yield for AF detection after stroke $(3-5 \%)^{38}$.

The association between AF and cryptogenic stroke was strengthened by intensive monitoring using ICM $^{22-24}$. Continuous electrocardiographic monitoring with ICM has been shown to be superior to conventional follow-up in detecting AF in the population with cryptogenic stroke ${ }^{24,30}$.

Further studies demonstrated that long-term continuous electrocardiographic monitoring with ICMs is significantly more effective than intermittent monitoring strategies (24-hour, 48-hour, and 7-day Holters and monthly 24-hour Holters) for identifying AF in patients with previous cryptogenic stroke ${ }^{25,39}$.

Current guidelines suggest to consider implanted loop recorder in stroke to optimize detection of silent AF, but the use of prolonged monitoring after CS is currently left to physician discretion ${ }^{14,40}$.

Even though in the last decades, randomized and controlled studies had demonstrated the importance of cardiac monitoring via ICMs devices in cryptogenic stroke patients ${ }^{24,29,30}$, there are only a few real-life studies with follow-up longer than 12 months. In a recent study by Ziegler et al. ${ }^{32} \mathrm{AF}$ was diagnosed in $21.5 \%$ of CS patients by 24 -months follow-up. Our study represents the first real-life cohort of CS patients in Europe with a follow-up longer more than 24 months.

Previous studies reported that the percentages of AF were $16 \%$ at 30 days and $21 \%$ at 2 years in a population of $280^{41}$ and $1247^{32}$ patients with a recent CS, respectively. At 24 months follow-up, we found a significantly higher DR of AF (41\%) in comparison with previous literature $\left(\chi^{2}=6.4 p=0.01 \text { and } \chi^{2}=4.3 p=0.04\right)^{32,41}$. This result has to be interpreted in light of several considerations. We suppose that $\mathrm{CHA}_{2} \mathrm{DS}_{2}$-VASc score, which estimates the risk of stroke in patients with $\mathrm{AF}^{17}$, may contribute to explain such a high overall DR in our cohort. In accord with previous studies, $\mathrm{CHA}_{2} \mathrm{DS}_{2}$-VASc score was higher in the AF than in the no-AF group $(\mathrm{p}=0.035)$, confirming that thromboembolic risk correlates with AF risk. Moreover, the higher frequency of previous strokes in the AF group can be correlated to the embolic etiology predisposing to recurrence. Then, previous stroke can be considered as a marker of higher risk for AF in patients with CS.

We hypothesize, indeed, that the age can influence the higher value of $\mathrm{CHA}_{2} \mathrm{DS}_{2}$-VASc score in the AF group. Our data showed that AF group was older than no-AF group $(\mathrm{p}=0.06)$, confirming the role of age on AF detection $^{42,43}$. In addition, patients from our cohort (68.1 \pm 9.3 years) are older than patients from previous literature (i.e. Ziegler's cohort, DR $21.5 \%$ at 24 months, mean age $65.3 \pm 13$ years; Sanna's cohort, DR $8.9 \%$ at six-month, mean age $61.6 \pm 11.4$ years, etc ${ }^{24,32}$; similarly, in Gladstone's cohort the DR and mean age were higher respect to the Sanna's cohort (DR $16.1 \%$ at three months, mean age $72.5 \pm 8.5$ years $)^{24,41}$. These considerations, taken together with our data, support the hypothesis that the occurrence of AF in CS patients was influenced by age. Unfortunately, previous studies used different methods for AF detection: in the study of Gladstone ${ }^{41} \mathrm{AF}$ was defined when it was longer than 30 seconds while other authors defined AF episodes lasting at least 2 minutes ${ }^{32}$. This issue may partially explain differences in the DR.

Interestingly, in our study LAVI was higher in the AF then in the no-AF group. LAVI has been reported to be associated with the risk of AF and then it might be useful for identifying individuals with a high risk of AF and thromboembolic complications, improving treatment outcomes ${ }^{44,45}$. Atrial disease or myopathy forms the substrate for AF and underlies the potential for atrial thrombus formation and subsequent stroke.

Also, in our study at 6 months follow-up DR was higher (22\%) than in a previous one $(8.9 \%)^{24}$. Even if we hypothesize that the age is the major contributor to the difference in DR among studies, it cannot be excluded that the shorter time interval between stroke and ICM placement in our cohort might have contributed to a higher DR. We suppose that a considerable percentage of arrhythmic events occurs in the early stages of stroke and may be lost by a delay in positioning the device. Finally, a selection bias could have reduced DR scores in previous studies that included patients with transient ischemic attacks ${ }^{24,41}$. Many diseases can mimic transient ischemic attacks ${ }^{46}$, thus generating a possible enrolment of patients who may not have vascular disease.

In the present real-world study, we show a high incidence of AF detected by ICM in a cohort of 58 CS patients consecutively recruited and followed for 3 years. Interestingly, our data support the idea that ICM can detect AF in CS patients after a complete diagnostic workup. Also, we provide evidence of the efficacy of anticoagulation in the AF group, as none had a recurrence of stroke, at the difference with one patient in the no-AF group. 
Moreover, AF was detected within 6 months after CS in 54\% of AF group and within 12 months in further 13\% (overall $70 \%$ at 12 months) with a growing DR in the first 24 months from ICM positioning (Fig. 1).

Finally, we found that patients in the AF group had higher frequency of previous strokes and higher $\mathrm{CHA}_{2} \mathrm{DS}_{2}$-VASc scores, as well as atrial volumes detected by transthoracic echocardiography. These data confirm that patients with a more severe cardiovascular profile are more prone to develop AF and arise the possibility of a connection between "atrial disease" and AF. However, more data are needed to deepen these relations.

A last consideration should be made about the prescription of anticoagulants in silent AF, as nowadays it is widely discussed. In our cohort, all patients had silent AF with single or multiple episodes of AF with a high AF burden, and all patients had high risk according to European Guidelines, allowing a full indication for oral anticoagulants ${ }^{47}$; moreover, we recorded lower stroke recurrence rates in the AF respect to the No-AF group ( 0 versus $3 \%$ after 30 months of follow-up), supporting the prescription of anticoagulants.

Limitations. Some technical issues should be pointed out: the device used in the study requires at least 2 minutes of AF to be detected and therefore episodes less than 2 minutes are not detected. On the contrary, many artifacts can mimic AF with a possibility of false positives; however, the confirmation of the diagnosis through an expert cardiologist can contain this possibility.

The most important limitation of this study is the sample size. Indeed, even if implantation of ICM was proposed to all patients with CS arriving in our stroke unit, some patients refused the implantation due to the invasiveness of the procedure. It would be interesting to reproduce our result in a larger cohort of CS patients.

This is an open label prospective study without comparison with other forms of monitoring; future studies should compare ICMs with other types of continuous or intermittent monitoring. Another limitation emerges from the time interval between the stroke and the device implantation that appears to be in an apparent delay in the no-AF group respect to the AF group, even if this delay was not statistically significant; this might have reduced the DR in the AF group, representing a factor that leads to underestimation of DR.

Further studies are needed to establish the role of ICM in CS in real-world populations.

\section{Conclusions}

This study describes an AF DR of $41 \%$ after 30 months in patients with a previous negative standard intermittent monitoring, thus reinforcing literature data on long-term monitoring of CS patients with the use of ICMs. Moreover, the study shows that CS patients with previous strokes, high $\mathrm{CHA}_{2} \mathrm{DS}_{2}$-VASc scores and high atrial volumes are more likely to have or develop AF during the follow-up; this subgroup of CS patients are ideal candidate for ICM implantation.

Received: 30 August 2019; Accepted: 24 January 2020;

Published online: 24 February 2020

\section{References}

1. Kolominsky-Rabas, P., Weber, M., Gefeller, O., Neundoerfer, B. \& Heuschmann, P. Epidemiology of Ischemic Stroke Subtypes According to TOAST Criteria. Stroke 32, 2735-2740 (2001).

2. Grau, A. et al. Risk Factors, Outcome, and Treatment in Subtypes of Ischemic Stroke: The German Stroke Data Bank. Stroke 32, 2559-2566 (2001).

3. Schulz, U. \& Rothwell, P. Differences in Vascular Risk Factors Between Etiological Subtypes of Ischemic Stroke. Stroke 34, 2050-2059 (2003).

4. Adams, H. et al. Classification of subtype of acute ischemic stroke. Definitions for use in a multicenter clinical trial. TOAST. Trial of Org 10172 in Acute Stroke Treatment. Stroke 24, 35-41 (1993).

5. Hart, R. et al. Embolic strokes of undetermined source: the case for a new clinical construct. The Lancet Neurology 13, 429-438 (2014).

6. Saver, J. Cryptogenic Stroke. New England Journal of Medicine 374, 2065-2074 (2016)

7. Dalen, J. \& Alpert, J. Silent Atrial Fibrillation and Cryptogenic Strokes. The American Journal of Medicine 130, $264-267$ (2017).

8. Wolf, P., Dawber, T., Thomas, H. \& Kannel, W. Epidemiologic assessment of chronic atrial fibrillation and risk of stroke: The fiamingham Study. Neurology 28, 973-973 (1978).

9. Petersen, P. \& Godtfredsen, J. Embolic complications in paroxysmal atrial fibrillation. Stroke 17, 622-626 (1986).

10. Dalen, J. Prevention of Embolic Strokes. Chest 141, 294-299 (2012).

11. Tsang, T. et al. The prevalence of atrial fibrillation in incident stroke cases and matched population controls in Rochester, Minnesota. Journal of the American College of Cardiology 42, 93-100 (2003).

12. Strickberger, S. et al. Relationship between atrial tachyarrhythmias and symptoms. Heart Rhythm 2, 125-131 (2005).

13. Hart, R., Pearce, L. \& Aguilar, M. Meta-analysis: Antithrombotic Therapy to Prevent Stroke in Patients Who Have Nonvalvular Atrial Fibrillation. Annals of Internal Medicine 146, 857 (2007).

14. Kirchhof, P. et al. 2016 ESC Guidelines for the management of atrial fibrillation developed in collaboration with EACTS. Kardiologia Polska, 1359-1469 (2016).

15. Jauch, E. et al. Guidelines for the Early Management of Patients With Acute Ischemic Stroke. Stroke 44, 870-947 (2013).

16. Hart, R. et al. Lessons from the Stroke Prevention in Atrial Fibrillation Trials. Annals of Internal Medicine 138, 831 (2003).

17. Lip, G., Nieuwlaat, R., Pisters, R., Lane, D. \& Crijns, H. Refining Clinical Risk Stratification for Predicting Stroke and Thromboembolism in Atrial Fibrillation Using a Novel Risk Factor-Based Approach. Chest 137, 263-272 (2010).

18. Healey, J. et al. Subclinical Atrial Fibrillation and the Risk of Stroke. New England Journal of Medicine 366, 120-129 (2012).

19. Flaker, G. et al. Asymptomatic atrial fibrillation: Demographic features and prognostic information from the Atrial Fibrillation Follow-up Investigation of Rhythm Management (AFFIRM) study. American Heart Journal 149, 657-663 (2005).

20. Ziegler, P., Koehler, J. \& Mehra, R. Comparison of continuous versus intermittent monitoring of atrial arrhythmias. Heart Rhythm 3, 1445-1452 (2006).

21. Charitos, E. et al. A Comprehensive Evaluation of Rhythm Monitoring Strategies for the Detection of Atrial Fibrillation Recurrence. Circulation 126, 806-814 (2012).

22. Etgen, T., Hochreiter, M., Mundel, M. \& Freudenberger, T. Insertable Cardiac Event Recorder in Detection of Atrial Fibrillation After Cryptogenic Stroke. Stroke 44, 2007-2009 (2013).

23. Dion, F. et al. Unexpected low prevalence of atrial fibrillation in cryptogenic ischemic stroke: a prospective study. Journal of Interventional Cardiac Electrophysiology 28, 101-107 (2010). 
24. Sanna, T. et al. Cryptogenic Stroke and Underlying Atrial Fibrillation. New England Journal of Medicine 370, 2478-2486 (2014).

25. Ritter, M. et al. Occult Atrial Fibrillation in Cryptogenic Stroke. Stroke 44, 1449-1452 (2013).

26. Cotter, P. et al. Incidence of atrial fibrillation detected by implantable loop recorders in unexplained stroke. Neurology 80, 1546-1550 (2013).

27. Rojo Martínez, E. et al. Alto rendimiento del holter implantable en la detección de fibrilación auricular paroxística oculta en pacientes con ictus criptogénico y sospecha de mecanismo embólico. Revista de Neurología 57, 251 (2013).

28. Christensen, L. et al. Paroxysmal atrial fibrillation occurs often in cryptogenic ischaemic stroke. Final results from the SURPRISE study. European Journal of Neurology 21, 884-889 (2014).

29. Sinha, A. et al. Cryptogenic Stroke and underlying Atrial Fibrillation (CRYSTAL AF): Design and rationale. American Heart Journal 160, 36-41 (2010).

30. Brachmann, J. et al. Uncovering Atrial Fibrillation Beyond Short-Term Monitoring in Cryptogenic Stroke Patients. Circulation: Arrhythmia and Electrophysiology, 9 (2016).

31. Elijovich, L., Josephson, S., Fung, G. \& Smith, W. Intermittent Atrial Fibrillation May Account for a Large Proportion of Otherwise Cryptogenic Stroke: A Study of 30-Day Cardiac Event Monitors. Journal of Stroke and Cerebrovascular Diseases 18, 185-189 (2009).

32. Ziegler, P. et al. Long-term detection of atrial fibrillation with insertable cardiac monitors in a real-world cryptogenic stroke population. International Journal of Cardiology 244, 175-179 (2017).

33. Ziegler, P. et al. Detection of Previously Undiagnosed Atrial Fibrillation in Patients With Stroke Risk Factors and Usefulness of Continuous Monitoring in Primary Stroke Prevention. The American Journal of Cardiology 110, 1309-1314 (2012).

34. Seet, R., Friedman, P. \& Rabinstein, A. Prolonged Rhythm Monitoring for the Detection of Occult Paroxysmal Atrial Fibrillation in Ischemic Stroke of Unknown Cause. Circulation 124, 477-486 (2011).

35. Rizos, T. et al. Continuous Stroke Unit Electrocardiographic Monitoring Versus 24-Hour Holter Electrocardiography for Detection of Paroxysmal Atrial Fibrillation After Stroke. Stroke 43, 2689-2694 (2012).

36. Douen, A., Pageau, N. \& Medic, S. Serial Electrocardiographic Assessments Significantly Improve Detection of Atrial Fibrillation 2.6-Fold in Patients With Acute Stroke. Stroke 39, 480-482 (2008).

37. Bhatt, A. et al. Predictors of Occult Paroxysmal Atrial Fibrillation in Cryptogenic Strokes Detected by Long-Term Noninvasive Cardiac Monitoring. Stroke Research and Treatment 2011, 1-5 (2011)

38. Liao, J., Khalid, Z., Scallan, C., Morillo, C. \& O’Donnell, M. Noninvasive Cardiac Monitoring for Detecting Paroxysmal Atrial Fibrillation or Flutter After Acute Ischemic Stroke. Stroke 38, 2935-2940 (2007).

39. Choe, W. et al. A Comparison of Atrial Fibrillation Monitoring Strategies After Cryptogenic Stroke (from the Cryptogenic Stroke and Underlying AF Trial). The American Journal of Cardiology 116, 889-893 (2015).

40. January, C. et al. 2019 AHA/ACC/HRS focused update of the 2014 AHA/ACC/HRS guideline for the management of patients with atrial fibrillation. Heart Rhythm 16, e66-e93 (2019).

41. Gladstone, D. et al. Atrial Fibrillation in Patients with Cryptogenic Stroke. New England Journal of Medicine 370, 2467-2477 (2014).

42. Samol, A., Hahne, K. \& Mönnig, G. Atrial fibrillation and silent stroke: links, risks, and challenges. Vascular Health and Risk Management, 65 (2016).

43. Wetterslev, M. et al. New-onset atrial fibrillation in adult critically ill patients: a scoping review. Intensive Care Medicine 45, 928-938 (2019).

44. Tsang, T. et al. Left atrial volume: important risk marker of incident atrial fibrillation in 1655 older men and women. Mayo Clinic Proceedings 76, 467-475 (2001)

45. Goldberger, J. et al. Evaluating the Atrial Myopathy Underlying Atrial Fibrillation. Circulation 132, 278-291 (2015).

46. Nadarajan, V., Perry, R., Johnson, J. \& Werring, D. Transient ischaemic attacks: mimics and chameleons. Practical Neurology 14, 23-31 (2014)

47. Kirchhof, P. et al. 2016 ESC Guidelines for the Management of Atrial Fibrillation Developed in Collaboration With EACTS. Rev Esp Cardiol (Engl Ed). 2017 Jan, 70, 50 (2017).

\section{Author contributions}

Dr. De Angelis conception, design of the study, collection of data, data analysis. Dr. Di Stefano conception, data analysis, writing and organization of the paper, tables and figures. Dr. Faustino collection of data, data analysis; Dr. Raffaella Franciotti manuscript preparation and statistical analysis. Dr. Furia, Dr. Di Girolamo and Prof. Onofrj review and critique.

\section{Competing interests}

The authors declare no competing interests.

\section{Additional information}

Correspondence and requests for materials should be addressed to V.D.S.

Reprints and permissions information is available at www.nature.com/reprints.

Publisher's note Springer Nature remains neutral with regard to jurisdictional claims in published maps and institutional affiliations.

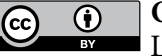

Open Access This article is licensed under a Creative Commons Attribution 4.0 International

License, which permits use, sharing, adaptation, distribution and reproduction in any medium or format, as long as you give appropriate credit to the original author(s) and the source, provide a link to the Creative Commons license, and indicate if changes were made. The images or other third party material in this article are included in the article's Creative Commons license, unless indicated otherwise in a credit line to the material. If material is not included in the article's Creative Commons license and your intended use is not permitted by statutory regulation or exceeds the permitted use, you will need to obtain permission directly from the copyright holder. To view a copy of this license, visit http://creativecommons.org/licenses/by/4.0/.

(C) The Author(s) 2020 